\title{
A Hybrid Finite-Volume/Transported PDF Model for Simulations of Turbulent Flames on Vector Machines
}

\author{
Stefan Lipp ${ }^{1}$, Ulrich Maas ${ }^{1}$, and Peter Lammers ${ }^{2}$ \\ 1 University Karlsruhe, Institute for Technical Thermodynamics, \\ lipp,mass@itt.mach.uni-karlsruhe.de \\ 2 University Stuttgart, High Performance Computing Center Stuttgart \\ lammers@hlrs.de
}

\section{Abstract}

The mathematical modeling of turbulent flames is a difficult task due to the intense coupling between turbulent transport processes and chemical kinetics. The mathematical model presented in this paper is focused on the turbulencechemistry interaction. The method consists of two parts. Chemical kinetics are taken into account a with reduced chemical reaction mechanism, which has been developed using the ILDM-Method ("Intrinsic Low-Dimensional Manifold"). The turbulence-chemistry interaction is described by solving the joint probability density function (JPDF) of velocity and scalars. Simulations of test cases with simple geometries verify the developed model.

\section{Introduction}

Reliable predictive models of turbulent flames are important in many industrial applications, e.g. the design and improvement of industrial burners used in gas turbines [1]. In particular new emission goals and new demands concerning fuel efficiency require detailed models which can treat the combustion chemistry without using oversimplified models. The standard methods for non-reacting flows (RANS, LES) cannot satisfactorily tackle the problem of the strong non-linearity of the chemical source term and often suffer from a poor modeling of the turbulence-chemistry interaction. However, a detailed modeling of this effect is possible for instance by applying probability density function methods (PDF). They show a high capability for modeling turbulent flames because these methods treat convection and finite rate non-linear chemistry exactly $[2,3]$. Only the effect of molecular mixing has to be modeled $[4,5,6]$. 
Two basically different PDF approaches exist in the literature . Some authors use stand-alone PDF models in which all hydrodynamic and thermokinetic properties of the flow are computed from a joint probability density function $[7,8,9,10]$. Other authors use the PDF transport equation only to calculate a certain number of the hydrodynamic and thermokinetic properties and calculate the remainder of the properties with an ordinary CFD solver [11, 12, 13]. In order to obtain the propability density function the repective transport equation must be solved. It can be derived from the Navier-Stokes equations [2]. As previously mentioned the chemistry term together with the body forces and the mean pressure gradient appear already in closed form here, only the terms describing the fluctuating pressure gradient and molecular mixing need to be modeled. Numerically the treatment of the PDF transport equation is quite different from the Navier Stokes equations. In contrast to the system of partial differential equations which is formed by the Navier-Stokes equations the transport equation for the PDF is a high dimensional scalar transport equation. In general it has $7+n_{S}$ dimensions which consist of three dimensions in space, three dimensions in velocity space, the time and the number of species $n_{S}$ used for the description of the thermokinetic state. Because of its high dimensionality it is not feasible to solve the equation using finitedifference or finite-volume methods. For that reason Monte Carlo methods have been employed, which are widely used in computational physics to solve problems of high dimensionality, because the numerical effort increases only linearly with the number of dimensions.

This solution method takes advantage of the fact that the PDF can be represented as an ensemble of stochastic particles [14]. The transport equation for the PDF is transformed to a system of stochastic ordinary differential equations. This system is constructed in such a way that the particle properties, e.g. velocity, scalars, and turbulent frequency, represent the same PDF as in the turbulent flow. In order to fulfill consistency of the modeled PDF, the mean velocity field derived from an ensemble of particles needs to satisfy the mass conservation equation [2]. This requires the pressure gradient to be calculated from a Poission equation. The available Monte Carlo methods cause strong bias solving a Poisson equation. This leads to stability problems calculating the pressure gradient. Different methods to calculate the mean pressure gradient where used in order to avoid these instabilities. One possibility is to couple the particle method with an ordinary finite-volume or finite-difference solver to obtain the mean pressure field from the Navier-Stokes equations. These so called hybrid PDF/CFD methods are widely used by different authors for many types of flames $[15,16,17,18,19,20]$.

In the presented paper a hybrid scheme is presented and used as well. The fields for mean pressure gradient and a turbulence charactaristic, e.g. the turbulent time scale, are derived solving the Favre averaged conservation equations for momentum and mass for the flow field using a finite-volume method. The effect of turbulent fluctuations is modeled using a $k-\tau$ model [21]. Chemical kinetics is taken into account by using the ILDM method to get reduced 
chemical mechanisms $[22,23]$. In the presented case the reduced mechanism describes the reaction with two parameters which on the one hand are few enough to limit the simulation time to an acceptable extent and on the other hand sufficient to get a detailed description of the chemical reaction.

The test case for the developed model is a model combustion chamber investigated by serveral authors $[24,25,26,27]$. With their data the results of the presented simulations are validated.

\section{Numerical Model}

The numerical model used is this paper is a hybrid CFD/PDF model and consists of two parts. A finite-volume solver for the Navier-Stokes equations (CFD) which provides the hydrodynamic quantities and a Monte Carlo solver for the probability density function (PDF) which gives the thermokinetic state of the flow. The principles of the solution procedure shall be briefly overviewed before going into details and discussing consistency and numerical matters. A sketch of the method is shown in Fig. 1. The calculation starts with a CFD step in which the Navier-Stokes equations for the flow flield are solved. As an intermediate result the current pressure gradient together with the mean velocities and the turbulence characteristics is handed over to the PDF part. Here the transport equation for the joint probability density function of the scalars describing the thermokinetic state and the velocities is solved. The result of the previous CFD step is considered at this point. The change of the thermokinetic state due to chemical reactions is calculated from a lookup table (ILDM). This table is based on a detailed chemical reaction mechanism which was in a preprocessing step reduced using the ILDM method. As a result of the PDF step the new mean molar mass and the mean temperature are returned to the CFD part. These internal iteration steps are performed until global convergence is achieved.

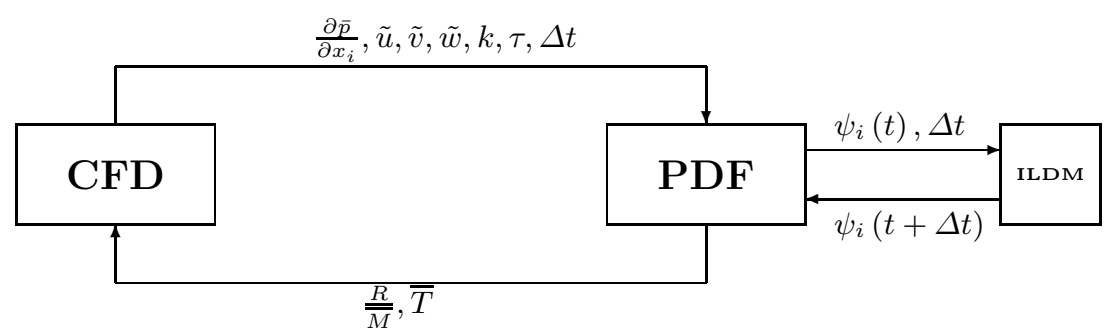

Fig. 1. Scheme of the coupling of CFD and PDF 


\subsection{CFD Model}

The utilized CFD code SPARC is an inhouse development of the Department of Fluid Machinery at Karlsruhe University [28]. It is a finite volume based solver for the compressible Navier-Stokes equations on block structured domains. Several turbulence closure models are implemented in this code. In the presented work a two equation model is applied which solves a transport equation for the turbulent kinetic energy and a turbulent time scale [21]. This model was selected due to being a good compromise in modeling accuracy and numerical stability.

\section{Navier-Stokes equations}

The system of partial differential equations which are solved are the mass conservation equation and the momentum conservation equation. All quanities appear in Favre-averaged manner. The Favre-average of any quantity $\zeta$ is calculated according to

$$
\tilde{\zeta}=\frac{\overline{\rho \cdot \zeta}}{\bar{\rho}}
$$

In detail the equations read

$$
\begin{aligned}
\frac{\partial \bar{\rho}}{\partial t}+\frac{\partial\left(\bar{\rho} \tilde{u}_{i}\right)}{\partial x_{i}} & =0 \\
\frac{\partial\left(\bar{\rho} \tilde{u}_{i}\right)}{\partial t}+\frac{\partial}{\partial x_{j}}\left(\bar{\rho} \tilde{u}_{i} \tilde{u}_{j}+\overline{\rho u_{i}^{\prime \prime} u_{j}^{\prime \prime}}+\bar{p} \delta_{i j}-\bar{\tau}_{i j}\right) & =0
\end{aligned}
$$

which are the conservation equations for mass and momentum in Favre average notation, respectively. In this model the solution of the energy equation is not necessary since the mean temerature is calculated in the PDF part from the variables desribing the thermokinetic state.

\section{Turbulence model}

The unclosed cross correlation term $\overline{\rho u_{i}^{\prime \prime} u_{j}^{\prime \prime}}$ in the momentum conservation equation is modeled using the Boussinesq approximation

$$
\overline{\rho u_{i}^{\prime \prime} u_{j}^{\prime \prime}}=\bar{\rho} \mu_{T}\left(\frac{\partial \tilde{u}_{i}}{\partial x_{j}}+\frac{\partial \tilde{u}_{j}}{\partial x_{i}}\right)
$$

The turbulent viscosity $\mu_{T}$ is given by a two equation model of Speziale et al. [21] with

$$
\mu_{T}=C_{\mu} f_{\mu} k \tau
$$

The parameter $C_{\mu}$ is an empirical constant with a value of $C_{\mu}=0.09$ and $f_{\mu}$ accounts for the influence of walls. The turbulent kinetic energy $k$ and the 
turbulent time scale $\tau$ are calculated from their transport equation which are [21]

$$
\begin{gathered}
\bar{\rho} \frac{\partial k}{\partial t}+\bar{\rho} \tilde{u}_{j} \frac{\partial k}{\partial x_{j}}=\tau_{i j} \frac{\partial \tilde{u}_{i}}{\partial x_{j}}-\bar{\rho} \frac{k}{\tau}+\frac{\partial}{\partial x_{i}}\left[\left(\mu+\frac{\mu_{T}}{\sigma_{k}}\right) \frac{\partial k}{\partial x_{j}}\right] \\
\bar{\rho} \frac{\partial \tau}{\partial t}+\bar{\rho} \tilde{u}_{j} \frac{\partial \tau}{\partial x_{j}}=\left(1-C_{\epsilon 1}\right) \frac{\tau}{k} \tau_{i j} \frac{\partial \tilde{u}_{i}}{\partial x_{j}}+\left(C_{\epsilon 2}-1\right) \bar{\rho}+ \\
\frac{\partial}{\partial x_{j}}\left[\left(\mu+\frac{\mu_{T}}{\sigma_{\tau 2}}\right) \frac{\partial k}{\partial x_{j}}\right]+\frac{2}{k}\left(\mu+\frac{\mu_{T}}{\sigma_{\tau 1}}\right) \frac{\partial k}{\partial x_{k}} \frac{\partial \tau}{\partial x_{k}}- \\
\frac{2}{\tau}\left(\mu+\frac{\mu_{T}}{\sigma_{\tau 2}}\right) \frac{\partial \tau}{\partial x_{k}} \frac{\partial \tau}{\partial x_{k}} .
\end{gathered}
$$

Here $C_{\epsilon 1}=1.44$ and $\sigma_{\tau 1}=\sigma_{\tau 2}=1.36$ are empirical model constants. The parameter $C_{\epsilon 2}$ is calculated from the turbulent Reynolds number $\mathrm{Re}_{\mathrm{t}}$.

$$
\begin{aligned}
\mathrm{Re}_{\mathrm{t}} & =\frac{k \tau}{\mu} \\
C_{\epsilon 2} & =1.82\left[1-\frac{2}{9} \exp \left(\left(-\mathrm{Re}_{\mathrm{t}} / 6\right)^{2}\right)\right]
\end{aligned}
$$

\subsection{Joint PDF Model}

In the literature many different joint PDF models can be found, for example models for the joint PDF of velocity and composition [29, 30] or for the joint PDF of velocity, composition and turbulent frequency [31]. A good overview of the different models can be found in [17].

In the work presented here a joint PDF of velocity and composition vector is employed. This is a one-time, one-point joint probability density function which has the main advantage to treat chemical reactions exactly without any modeling assumptions [2]. However, the effect of molecular mixing has to be modeled.

\section{Joint Probability Density Function}

The state of a reacting fluid flow at one point in space and time can be fully described by the velocity vector $\mathbf{V}=\left(V_{1}, V_{2}, V_{3}\right)^{T}$ and the composition vector $\Psi$ containing the mass fractions of $n_{S}-1$ species and the enthalpy $h$ $\left(\boldsymbol{\Psi}=\left(\Psi_{1}, \Psi_{2}, \ldots, \Psi_{n_{s}-1}, h\right)^{T}\right)$. The joint probability density function is defined as that function which gives, when integrated by the hole state space, the probability that at one point in space and time one realization of the flow falls within the interval

$$
\mathbf{V} \leq \mathbf{U} \leq \mathbf{V}+\mathrm{d} \mathbf{V}
$$

for its velocity vector and 


$$
\boldsymbol{\Psi} \leq \boldsymbol{\Phi} \leq \boldsymbol{\Psi}+\mathrm{d} \boldsymbol{\Psi}
$$

for its composition vector. Thus the the joint PDF reads

$$
f_{\mathbf{U} \phi}(\mathbf{V}, \boldsymbol{\Psi} ; \mathbf{x}, t) \mathrm{d} \mathbf{V} \mathrm{d} \boldsymbol{\Psi}=\operatorname{Prob}(\mathbf{V} \leq \mathbf{U} \leq \mathbf{V}+\mathrm{d} \mathbf{V}, \Psi \leq \boldsymbol{\Phi} \leq \boldsymbol{\Psi}+\mathrm{d} \boldsymbol{\Psi})
$$

\section{PDF Transport Equation}

According to [2] a transport equation can be derived for the joint PDF of velocity and composition. Under the assumption that the effect of pressure fluctuations on the fluid density is negligible the transport equation writes

$$
\begin{aligned}
\underbrace{\rho(\boldsymbol{\Psi}) \frac{\partial \tilde{f}}{\partial t}}_{I} & +\underbrace{\rho(\boldsymbol{\Psi}) U_{j} \frac{\partial \tilde{f}}{\partial x_{j}}}_{I I}+\underbrace{\left.\left[\left(\rho(\boldsymbol{\Psi}) g_{j}-\frac{\partial\langle p\rangle}{\partial x_{j}}\right)\right]\right] \frac{\partial \tilde{f}}{\partial U_{j}}}_{I I I}+\underbrace{\frac{\partial}{\partial \Psi_{\alpha}}\left[\rho(\boldsymbol{\Psi}) S_{\alpha}(\boldsymbol{\Psi}) \tilde{f}\right]}_{I V} \\
& =\underbrace{\frac{\partial}{\partial U_{j}}\left[\left\langle-\frac{\partial \tau_{i j}}{\partial x_{i}}+\frac{\partial p^{\prime}}{\partial x_{i}} \mid \mathbf{U}, \boldsymbol{\Psi}\right\rangle \tilde{f}\right]}_{V}+\underbrace{\frac{\partial}{\partial \Psi_{\alpha}}\left[\left\langle-\frac{\partial J_{i}}{\partial x_{i}} \mid \mathbf{U}, \boldsymbol{\Psi}\right\rangle \tilde{f}\right]}_{V I} \cdot(13)
\end{aligned}
$$

Term I describes the instationary change of the PDF, Term II its change due to convection in physical space and Term III takes into account the influence of gravitiy and the mean pressure gradient on the PDF. Term IV includes the chemical source term which describes the change of the PDF in composition space due to chemical reactions. All terms on the left hand side of the equation appear in closed form, e.g. the chemical source term. In contrast, the terms on the right hand side are unclosed and need further modeling. Many closing assumptions for these two terms exist. In the following only the ones that are used in the present work shall be explained further.

Term V describes the influence of pressure fluctuations and viscous stresses on the PDF. Commonly a Langevin approach $[32,33]$ is used to close this term. In the presented case the SLM (Simplified Langevin Model) is used [2]. More sophisticated approaches that take into account the effect of non-isotropic turbulence or wall effects exist as well [32, 34]. But in the presented case of a free stream flame the closure of the term by the SLM is assumed to be adequate and was chosen because of its simplicity and numerical robustness.

Term VI regards the effect of molecular diffusion within the fluid. This diffusion flattens the steep composition gradients which are created by the strong vortices in a turbulent flow. The model uses in this work has originally been proposed by Curl [35], then been modified by Janika et al. [36] and Pope [37] and is used here in this modified form.

\section{Solution of the PDF Transport equation}

As previously mentioned it is numerically unfeasable to solve the PDF transport equation with finite-volume or finite-difference methods because of its 
high dimensionality. Therefore a Monte Carlo method is used to solve the transport equation making use of the fact that the PDF of a fluid flow can be represented as a sum of $\delta$-functions.

$$
f_{\mathbf{U}, \boldsymbol{\phi}}^{*}(\mathbf{U}, \boldsymbol{\Psi} ; \mathbf{x}, t)=\sum_{i=1}^{N(t)} \delta\left(\mathbf{v}-\mathbf{u}^{i}\right) \delta\left(\phi-\Psi^{i}\right) \delta\left(\mathbf{x}-\mathbf{x}^{i}\right)
$$

Utilizing this fact the PDF of the fluid flow can be disreticed by a set of stochastic particles. For these particles equations of motion in space, velocity space and compostion space can be derived. Now, instead of the high dimensional PDF transport equation a set of (stochastic) ordinary differential equations are solved. The evolution of the particle position $\mathbf{X}_{\mathbf{i}}^{*}$ reads

$$
\frac{\mathrm{d} \mathbf{X}_{\mathbf{i}}^{*}}{\mathrm{~d} t}=\mathbf{U}_{\mathbf{i}}^{*}(t)
$$

in which $\mathbf{U}_{\mathbf{i}}^{*}$ is the velocity vector for each particle.

The evolution of the particles in the velocity space can be calculated according to the Simplified Langevin Model [2] by

$$
\mathrm{d} U_{i}^{*}=-\frac{\partial \bar{p}}{\partial x_{i}} \mathrm{~d} t-\left(\frac{1}{2}+\frac{3}{4} C_{0}\right)\left[U_{i}^{*}-\left\langle U_{i}\right\rangle\right] \frac{\mathrm{d} t}{\tau}+\sqrt{\frac{C_{0} k}{\tau}} \mathrm{d} W_{i}
$$

Here the equation is only written for the $U$ component of the velocity vector $\mathbf{U}=(U, V, W)^{T}$ belonging to the spacial coordinate $x\left(\mathbf{x}=(x, y, z)^{T}\right)$ for reasons of simplicity. The equations of the other components $V, W$ look accordingly. In eq. $16 \partial \bar{p} / \partial x_{i}$ denotes the mean pressure gradient, $\left\langle U_{i}\right\rangle$ the mean particle velocity, $t$ the time, $\mathrm{d} W_{i}$ a differential Wiener increment, $C_{0}$ a model constant, $k$ and $\tau$ the turbulent kinetic energy and the turbulent time scale, respectively.

Finally the evolution of the composition vector can be calculated as

$$
\frac{\mathrm{d} \boldsymbol{\Psi}}{\mathrm{d} t}=S+M
$$

in which $S$ is the chemical source term (appearing in closed form) and $M$ denotes the effect of molecular mixing. This term is unclosed and as mentioned previously is modeled by a modified Curl model [36].

\section{Chemical Kinetics}

The source term appearing in eq. 17 is calculated from a lookup table which is created using automatically reduced chemical mechanisms. The deployed technique to create these tables is the ILDM method ("Intrinsic Low-Dimensional Manifold") by Maas and Pope [22, 23].

The basic idea of this method is the identification and separation of fast and 
slow time scales. In typical turbulent flames the time scales governing the chemical kinetics range from $10^{-9} \mathrm{~s}$ to $10^{2} \mathrm{~s}$. This is a much larger spectrum than that of the physical processes (e.g. molecular transport) which only vary from $10^{-1} \mathrm{~s}$ to $10^{-5} \mathrm{~s}$. Reactions that occur in the very fast chemical time scales are in partial equilibrium and the species are in steady state. These are usually responsible for equilibrium processes. Making use of this fact it is possible to decouple the fast time scales. The main advantage of decoupling the fast time scales is that the chemical system can be described with a much smaller number of variables (degrees of freedom).

In our test case the chemical kinetics are described with only two parameters namely the mixure fraction and the specific mole number of $\mathrm{CO}_{2}$ instead of the 34 species (degrees of freedom) appearing in the detailed methane reaction mechanism. Further details of the method and its implementation can be found in $[22,23]$.

\subsection{Coupling of the solution procedure}

The coupling of the CFD and the PDF part of the model is done with the calculation of the pressure via the equation of state in the CFD part which closes the equation system.

$$
\bar{p}=\bar{\rho} \cdot \frac{R}{\bar{M}} \cdot \bar{T}
$$

Here the mean temperature $\bar{T}$ is calculated (together with the mean molar mass $\bar{M}$ ) from the PDF solver instead of from the energy equation as done in standard compressible flow solvers. The density $\bar{\rho}$ is calculated in the CFD part from the mass conservation equation.

\section{Performance Optimization for SX-8}

In the Teraflop-Workbench project which is a collaboration between NEC and the HLRS we are optimizing the presented model, especially the PDF part, for the NEC SX architecture.

We started with significant changes in the data structure of the PDF solver. For instance, we now use modern Fortran language constructs to increase the solvers flexibility in handling the block structured grids of the CFD solver. This results in changes of the arrays holding the information of the stochastic particles as well as of the arrays for exchanging quantities between CFD and PDF solver etc..

At first some general remarks may help to understand the expected performance on the SX. As the code implements a hybrid method in the sense that some quantities are calculated by the CFD code and others by a PDF Monte-Carlo method one has to continuously exchange information between the quantities defined on the grid nodes of the CFD solver and the particles 
of the PDF part and vice versa. This requires the maintenance of list vectors in each time step which hold the relation between particle position and grid nodes/cells. Consequently, this results further in two kinds of loop structures appearing in the solver. For the first kind of loops in which the inner loop goes over all particles within the domain the vector length is always sufficient. On the other hand, there are a lot of cases in which the inner loop has to run over the particles located in one cell of the CFD solver grid. We currently use a number of particles per cell which lies around 100 and is therefore not sufficient for the SX-8. But, we will increase this number in the future as the quality of the statistics will improve naturally with a larger number of particles per cell. Then, the PDF part of the solver is likely to benefit from the SX architecture.

Beside changing the data structures we have already started to work on performance improvements of the algorithm for solving the joint PDF transport equation itself. It is done with a so called fractional step algorithm which consists of five steps:

$$
\frac{\partial \mathcal{F}}{\partial t}=P_{1}+P_{2}+P_{3}+P_{4}+P_{5}
$$

$P_{1}$ and $P_{4}$ deal with the convection of the particles in each time step. Each part applies eq. 15 for $\Delta t / 2$. With the discretization chosen so far we talk algorithmically about a daxpy loop for the three particle positions. In a two dimensional model the particles moving outside the plan have to be projected back into it. Now, everything is done in one collapsed loop over all particles. Additionally the routine consist of two debugging loops, which have been vectorized. Altogether the performance of $P_{1}$ and $P_{4}$ has been improved by a factor of more than 20 . The next step $P_{2}$ deals with the changes in the particles velocity by applying the simplified Langevin model eq. 16 . The corresponding loop is now vectorized over all particles. But, beforehand it is necessary to get several quantities from the flow solver and interpolate them to the particles position. This is an example for loops where the vector length is restricted to the number of particles inside a cell. So far, we achieved an improvement of a factor three in comparison to the original version. What remains to be done for $P_{2}$ is the vectorization of the Wiener increment determination (see below).

We conclude this section with some bullet points regarding our plans for future changes and improvements:

- $P_{2}$ : The currently used random number generators are not suitable for large scale computations. Therefore, they have to be substituted anyway. We will implement ASL (Advance Scientific Library) routines with a very long period. If necessary, the algorithms will be changed to enable vectorization (Wiener increment).

- $P_{3}$ : Vectorization of the mixing model. 
- $P_{5}$ The layout of the tables containing the chemical reactions will be changed. This should offer huge possibilities to improve the solvers performance.

- Improving the administrative part needed for coupling the PDF and the CFD parts.

- Parallel version of the PDF part.

\section{Results and Discussion}

Results of simulations of a simple swirling premixed confined turbulent flame are shown. Turbulent swirling flames which are aerodynamically stabilized by an internal recirculation zone were used in many industrial applications such as jet engines and gas turbines. However, some major physical effects appearing in these flames are still not entirely understood. That includes also effects like the combustion induced vortex breakdown or presizing vortex cores which might be critical with respect to a save operation. Parameter studies on test rigs with a simplified geometry should clarify the influence of global operation parameters like the equivalence ratio and the swirl number on these effects. Details of the test rig simulated in this work and the experimental data can be found in $[25,26,27]$. A validation of the flow field for the non-reacting case was subject of previous work. The results can be found in [38]. The results presented in this paper will therefore focus mainly on the reacting case. Due to a lack of experimental data only qualitative statements can be made. Fig. 2

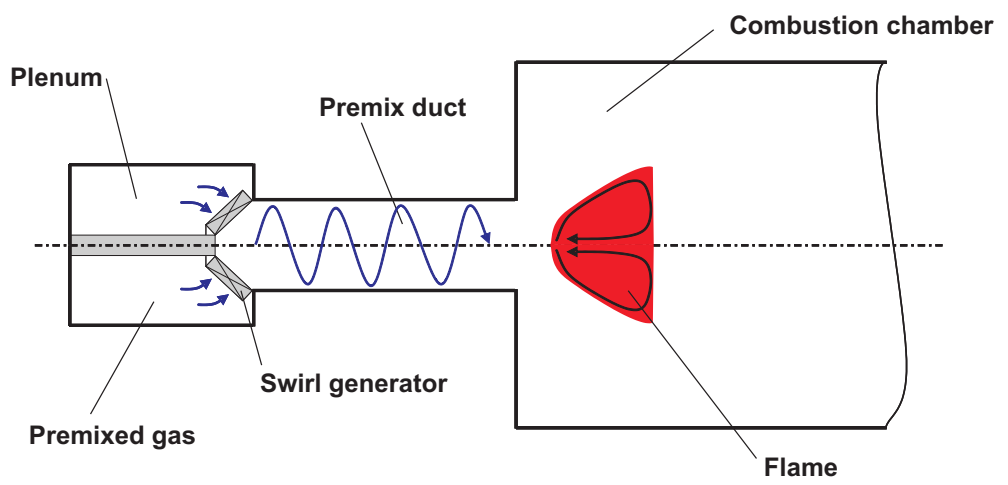

Fig. 2. Sketch of the investigated combustion chamber

shows a sketch of the test rig investigated. It consists of three parts: a plenum, the so-called premix duct and the combustion chamber. In the plenum a perfectly premixed methane-air mixture is aided through the swirl generator into the premixing duct. The swirling flow continues through the premix duct into the combustion chamber. Here a vortex breakdown occurs which creates the 
internal recirculation zone. There the flame is aerodynamically stabilized by hot exhaust gas which enables a save and stable operation of the hole burner. Fig. 3 shows the position of the computational domain with respect to the

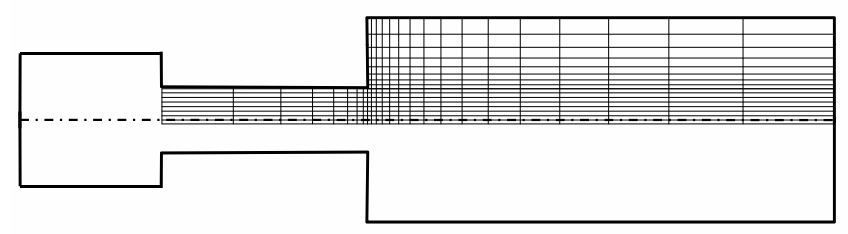

Fig. 3. Position of the computational domain

physical domain. Only the premixing duct and the combustion chamber are simulated. The spatial model is reduced to a $2 \mathrm{D}$ axisymmetric solution domain which makes parameter studies faster and more convenient. To account for the effect of the swirl generator profiles of all hydrodynamic properties are used as boundary conditions. These profiles are taken from the work of Kiesewetter et al. [24] and are derived from detailed simulations of the entire burner including the plenum and the swirl generator. The same author also shows that this mapping from $3 \mathrm{D}$ to $2 \mathrm{D}$ axisymmetric solution domain leads to reasonable results.

The global operation parameters are an equivalence ratio of $\phi=0.556$, a total inlet mass flux of $70 \mathrm{~g} / \mathrm{s}$ and a swirl number of $S=0.5$. The preheat temparture of the mixture is $T=298 \mathrm{~K}$.

All the results shown are for the reactive case. Fig. 4 depicts the axial component of the velocity vector. Shown is a contour plot spanning over the streamwise $(x)$ and radial $(y)$ coordinate in space. Even though the simulations are undertaken making use of the axial symmetry the results are mirrored at the symmetry line in the shown figures for reasons of clarity. The contour plot in fig. 4 shows two areas with negative axial velocity: One in the corner of the combustion chamber caused by the backward facing step and another on the symmetry line aerodynamically caused by the swirl in the main flow. This internal recirculation zone is used to stabilize the flame by holding hot exhaust gases.

This can be observed in fig. 5 as well. This figure shows the temperature field of the flame. The temperature as explained above is calculated from a lookup table. One can see that the reaction of the premixed fresh gas starts on the symmetry line where the hot exhaust gas is transported by the internal recirculation zone. A steep temperature gradient can be observed and the temperature after the flame front is resonably well predicted by the presented model.

An aspect of main interest in this studies is the stability of the flame under 


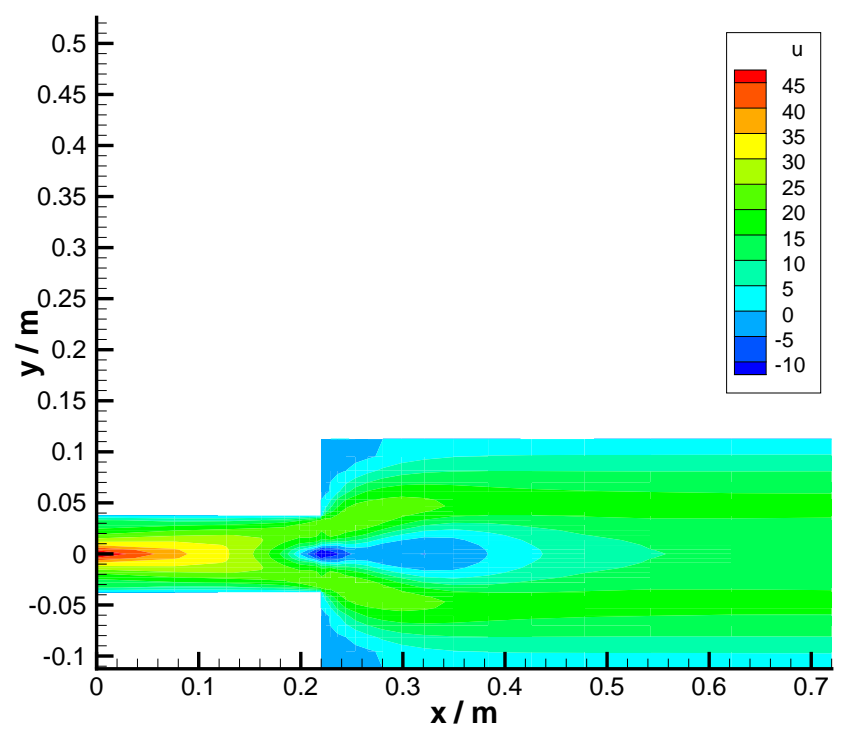

Fig. 4. Contourplot of the axial velocity component $\left(u / \frac{\mathrm{m}}{\mathrm{s}}\right)$

different global operation condition (e.g. different equivalence ratios). Therefore fig. 6 shows a closeup of the combustion chamber at the position of the backward facing step. The figures depicts again a contour plot. In the upper half the temperature field is shown while in the lower half the axial velocity can be seen. Additionally the $u=0 \mathrm{~m} / \mathrm{s}$ isoline is plotted. The isoline marks the boundary of the internal recirculation zone. One can see that the flame is located within the recirculation bubble and a slight increase of temperature due to prereactions can be observed close to the symmetry line in front of the bubble in regions of slow axial valocity. A stable operation of the flame is possible as long as the recirculation bubble is stationary in space.

\section{Conclusion}

Simulations of premixed swirling methane-air flames are presented. The model consists of two parts: a finite-volume solver for the hydrodynamic quantities and a Monte Carlo solver for the transport equation of the JPDF of velocity and scalars. This provides a detailed modeling of the turbulence chemistry interaction. Chemical kinetics are described by automatically reduced mechanisms created with the ILDM method.

The presented results show that a at least the qualitative behaviour of the 


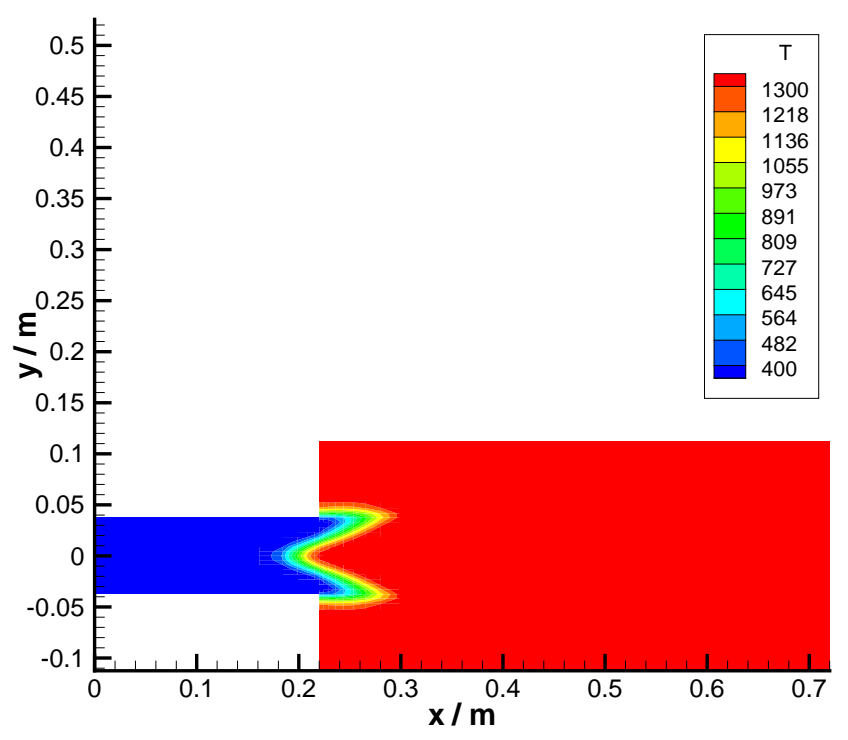

Fig. 5. Contourplot of the temperature $(T / K)$

flame could be predicted for the stationary case. Although quantitative comparison is not possible due to a lack of detailed experimental data. The potential of the model to predict turbulent flames quantitatively is currently investigated using for some test cases where large experimental data sets are available. An analysis of instationary effects like the combustion induced vortex breakdown which shall be trigged by a variation of the equivalence ratio is subject of future research work.

\section{Acknowlegment}

The simulations were performed on the national super computer NEC SX-8 at the High Performance Computing Center Stuttgart (HLRS) under the grant number FLASPARC. The work of Franco Magagnato (Department of Fluid Machinery, University Karlsruhe) suppling the CFD code SPARC and assisting with its use is gratefully acknowledged.

\section{References}

1. C. Duwig, D. Stankovic, L. Fuchs, G. Li, and E. Gutmark. Experimental and numerical study of flameless combustion in a model gas turbine combustor. 


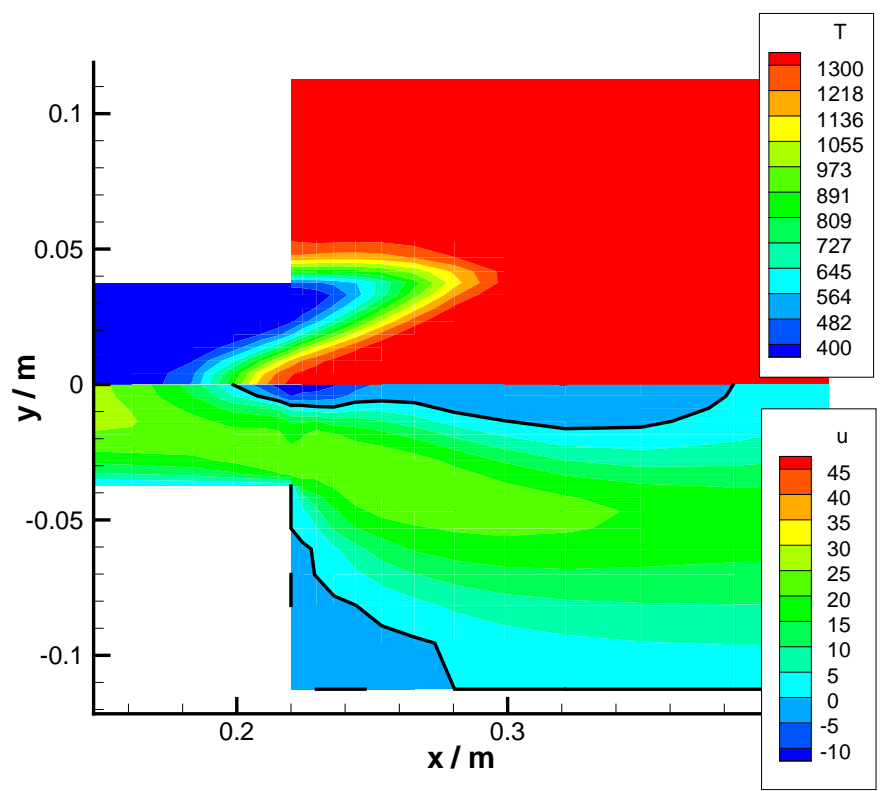

Fig. 6. Temperature and axial velocity

Combustion Science and Technology, 180(2):279-295, 2008.

2. S.B. Pope. Pdf methods for turbulent reactive flows. Progress in Energy Combustion Science, 11:119-192, 1985.

3. S.B Pope. Lagrangian pdf methods for turbulent flows. Annual Review of Fluid Mechanics, 26:23-63, 1994.

4. Z. Ren and S.B. Pope. An investigation of the performence of turbulent mixing models. Combustion and Flame, 136:208-216, 2004.

5. B. Merci, D. Roekaerts, B. Naud, and S.B. Pope. Comparative study of micromixing models in transported scalar pdf simulations of turbulent nonpremixed bluff body flames. Combustion and Flame, 145:109-130, 2006.

6. B. Merci, D. Roekaerts, and B. Naud. Study of the perfomance of three micromixing models in transported scalar pdf simulations of a piloted jet diffusion flame ("delft flame iii"). Combustion and Flame, 144:476-493, 2006.

7. P.R. Van Slooten and S.B Pope. Application of pdf modeling to swirling and nonswirling turbulent jets. Flow Turbulence and Combustion, 62(4):295-334, 1999.

8. V. Saxena and S.B Pope. Pdf simulations of turbulent combustion incorporating detailed chemistry. Combustion and Flame, 117(1-2):340-350, 1999.

9. S. Repp, A. Sadiki, C. Schneider, A. Hinz, T. Landenfeld, and J. Janicka. Prediction of swirling confined diffusion flame with a monte carlo and a presumedpdf-model. International Journal of Heat and Mass Transfer, 45:1271-1285, 2002 . 
10. K. Liu, S.B. Pope, and D.A. Caughey. Calculations of bluff-body stabilized flames using a joint probability density function model with detailed chemistry. Combustion and Flame, 141:89-117, 2005.

11. M. Nau. Berechnung turbulenter Diffusionsflammen mit Hilfe eines Verfahrens zur Bestimmung der Wahrscheinlichkeitsdichtefunktion und automatisch reduzierter Reaktionsmechanismen. PhD thesis, Universität Stuttgart, Fakult"at f"ur Energietechnik, 1997.

12. R. Bender, U. Maas, S. Böckle, J. Kazenwadel, C. Schulz, and J. Wolfrum. Monte-carlo-pdf-simulation and raman/rayleigh-measurement of a turbulent premixed flame. In Twenty-Eighth Symposium (International) on Combustion. The Combustion Institute, Pittsburgh, PA, 2000.

13. Y.Z. Zhang and D.C. Haworth. A general mass consistency algorithm for hybrid particle/finite-volume pdf methods. Journal of Computational Physics, 194:156193, 2004.

14. S.B Pope. A monte carlo method for pdf equations of turbulent reactive flow. Combustion, Science and Technology, 25:159-174, 1981.

15. P. Jenny, M. Muradoglu, K. Liu, S.B. Pope, and D.A. Caughey. Pdf simulations of a bluff-body stabilized flow. Journal of Computational Physics, 169:1-23, 2000.

16. A.K. Tolpadi, I.Z. Hu, S.M. Correa, and D.L. Burrus. Coupled lagrangian monte carlo pdf-cfd computation of gas turbine combustor flowfields with finite-rate chemistry. Journal of Engineering for Gas Turbines and Power, 119:519-526, 1997.

17. M. Muradoglu, P. Jenny, S.B Pope, and D.A. Caughey. A consistent hybrid finite-volume/particle method for the pdf equations of turbulent reactive flows. Journal of Computational Physics, 154:342-370, 1999.

18. M. Muradoglu, S.B. Pope, and D.A. Caughey. The hybid method for the pdf equations of turbulent reactive flows: Consistency conditions and correction algorithms. Journal of Computational Physics, 172:841-878, 2001.

19. G. Li and M.F. Modest. An effective particle tracing scheme on structured/unstructured grids in hybrid finite volume/pdf monte carlo methods. Journal of Computational Physics, 173:187-207, 2001.

20. V. Raman, R.O. Fox, and A.D. Harvey. Hybrid finite-volume/transported pdf simulations of a partially premixed methane-air flame. Combustion and Flame, 136:327-350, 2004.

21. H.S. Zhang, R.M.C. So, C.G. Speziale, and Y.G. Lai. A near-wall two-equation model for compressible turbulent flows. In Aerospace Siences Meeting and Exhibit, 30th, Reno, NV, page 23. AIAA, 1992.

22. U. Maas and S. B. Pope. Simplifying chemical kinetics: Intrinsic low-dimensional manifolds in composition space. Combustion and Flame, 88:239-264, 1992.

23. U. Maas and S.B. Pope. Implementation of simplified chemical kinetics based on intrinsic low-dimensional manifolds. In Twenty-Fourth Symposium (International) on Combustion, pages 103-112. The Combustion Institute, 1992.

24. F. Kiesewetter, C. Hirsch, J. Fritz, M. Kröner, and T. Sattelmayer. Twodimensional flashback simulation in strongly swirling flows. In Proceedings of ASME Turbo Expo 2003, 2003.

25. M. Kröner. Einfluss lokaler Löschvorgänge auf den Flammenrückschlag durch verbrennungsinduziertes Wirbelaufplatzen. $\mathrm{PhD}$ thesis, Technische Universität München, Fakultät für Maschinenwesen, 2003. 
26. J. Fritz. Flammenrückschlag durch verbrennungsinduziertes Wirbelaufplatzen. $\mathrm{PhD}$ thesis, Technische Universität München, Fakultät für Maschinenwesen, 2003.

27. F. Kiesewetter. Modellierung des verbrennungsinduzierten Wirbelaufplatzens in Vormischbrennern. PhD thesis, Technische Universität München, Fakultät für Maschinenwesen, 2005.

28. F. Magagnato. SpARC Structured PArallel Research Code. Department of Fluid Machinery, Karlsruhe University, Kaiserstrasse 12, 76131 Karlsruhe, Germany, 1998.

29. D.C. Haworth and S.H. El Tahry. Propbability density function approach for multidimensional turbulent flow calculations with application to in-cylinder flows in reciproating engines. AIAA Journal, 29:208, 1991.

30. S.M. Correa and S.B. Pope. Comparison of a monte carlo pdf finite-volume mean flow model with bluff-body raman data. In Twenty-Fourth Symposium (International) on Combustion, page 279. The Combustion Institute, 1992.

31. W.C. Welton and S.B. Pope. Pdf model calculations of compressible turbulent flows using smoothed particle hydrodynamics. Journal of Computational Physics, 134:150, 1997.

32. D.C. Haworth and S.B. Pope. A generalized langevin model for turbulent flows. Physics of Fluids, 29:387-405, 1986.

33. H.A. Wouters, T.W. Peeters, and D. Roekaerts. On the existence of a generalized langevin model representation for second-moment closures. Physics of Fluids, 8(7):1702-1704, 1996.

34. T.D. Dreeben and S.B. Pope. Pdf/monte carlo simulation of near-wall turbulent flows. Journal of Fluid Mechanics, 357:141-166, 1997.

35. R.L. Curl. Dispersed phase mixing: 1. theory and effects in simple reactors. A.I.Ch.E. Journal, 9:175,181, 1963.

36. J. Janicka, W. Kolbe, and W. Kollmann. Closure of the transport equation of the probability density function of turbulent scalar flieds. Journal of NonEquilibrium Thermodynamics, 4:47-66, 1979.

37. S.B Pope. An improved turbulent mixing model. Combustion, Science and Technology, 28:131-135, 1982.

38. S. Lipp and U. Maas. Simulations of premixed swirling flames using a hybrid finite-volume/transported pdf approach. In High Performance Computing on Vector Systems, pages 181-193, 2007. 